\title{
Compact Balanced Dual-Band Bandpass Filter Based on Modified Coupled-Embedded Resonators
}

\author{
Fulya Bagci, Member, IEEE, Armando Fernández-Prieto, Member, IEEE, Aintzane Lujambio, \\ Jesús Martel, Senior Member, IEEE, Joaquín Bernal, Member, IEEE, \\ and Francisco Medina, Fellow, IEEE
}

\begin{abstract}
A new compact balanced dual-band bandpass filter based on coupled-embedded resonators with modified ground plane is presented in this work. Common-mode is rejected within the two differential passbands by symmetrically introducing four coupled U-shaped defected ground structures below the resonators. Common-mode rejection is significantly improved when compared with the standard (solid ground plane) filter with similar geometry thanks to the introduction of four extra transmission zeros. Due to the symmetry, the differential mode is not significantly affected by the presence of the U-shaped resonators. Circuit-model data, full-wave simulations and measurements are provided to verify the benefits of the proposed dual-band filter.
\end{abstract}

Index Terms-Balanced filter, common-mode suppression, dual-band filter.

\section{INTRODUCTION}

$\mathbf{R}$ F/MICROWAVE balanced bandpass filters (BPFs) have attracted the interest of the microwave community in recent years due to their enhanced signal to noise ratio, noise immunity, low crosstalk and low electromagnetic interference (EMI) when compared with their classical single-ended counterparts. Nowadays, with the rapid growth of multiband wireless communication systems, multiband BPFs with very demanding specifications concerning both differential mode (DM) transmission and common-mode mode (CM) suppression are required. Several proposals regarding dual-band balanced BPFs can be found in the recent literature [1]-[9]. In [1]-[4], [7] balanced dual-band operation is obtained by means of electrically-coupled resonators. In such designs,

Manuscript received May 22, 2016; revised July 21, 2016; accepted October 10, 2016. Date of publication December 14, 2016; date of current version January 6, 2017. This work has been supported in part by the Spanish Ministerio de Economía y Competitividad with European Union FEDER Funds (contract TEC2013-41913-P), by the Spanish Junta de Andalucía (project P12-TIC-1435), and by TUBITAK, The Scientific and Technological Research Council of Turkey, under grant 22B2219

F. Bagci is with the Department of Electronics and Electromagnetism, Physics Faculty, University of Sevilla, Av. Reina Mercedes s/n, Seville 41012 Spain and also with the Department of Engineering Physics, Faculty of Engineering, Ankara University, Besevler, Ankara 06100, Turkey.

A. Fernández-Prieto and F. Medina are with the Department of Electronics and Electromagnetism, Physics Faculty, University of Sevilla, Av. Reina Mercedes s/n, Seville 41012, Spain (e-mail: armandof@us.es).

A. Lujambio is with the Skylife Engineering, Parque Tecnológico Aeroespacial de Andalucía, Early Ovington Street, 24, Nave 15-16, Seville 41309, Spain.

J. Martel is with the Department of Applied Physics II, ETS Architecture School, Av.Reina Mercedes s/n, Seville 41012, Spain.

J. Bernal is with the Dept. of Applied Physics III, ETS Ingenieros, University of Sevilla, Av. de los Descubrimientos, Seville 41092, Spain.

Color versions of one or more of the figures in this paper are available online at http://ieeexplore.ieee.org.

Digital Object Identifier 10.1109/LMWC.2016.2629962
CM suppression is improved by adding extra lumped capacitors, inductors, resistors and/or open-circuited stubs. Other recently proposed approaches to DM filter design with CM suppression make use of asymmetrical coupled lines [5] or substrate integrated waveguide (SIW) technology [8]. These designs provide good DM performance and CM rejection, but they suffer from the problem of having large electrical size and, moreover, the use of a large number of via-holes. In [6] balanced dual-band filtering is achieved by using coupled-embedded resonators. In this implementation the CM is rejected by cascading with the filter a pair of CM rejection differential-line stages based on the use of a low-pass DGS structure. Unfortunately, this solution increases the design complexity and the overall electrical size. Coupled complementary split-ring resonators (C-CSRR) have very recently been proposed [9] for the design of compact balanced dualband filters with good CM rejection. In this letter, a new compact balanced dual-band BPF based on coupled-embedded resonators with a modified ground plane is proposed. Design methodology and experimental validation of the structure are illustrated with a specific filter example operating at two wireless local area network (WLAN) bands. Measured and simulated results show the benefit of the proposed configuration.

\section{Proposed DGS Structure}

The layout of the proposed balanced dual-band BPF is shown in Fig.1. The top layer geometry is based on the singleended and differential dual-band filters reported in [10] and [6] respectively. It consists of the combination of two different sub-filters that enable the generation of two different DM passbands. These bands can be independently tuned. The bottom layer is formed by four U-shaped slot resonators. These resonators have been used in [11], [12] to improve the CM rejection of differential lines. In this letter the DGS resonators are embedded within the printed filter area, thus yielding a more compact design. When operating in DM, the $A A^{\prime}$ symmetry plane (see Fig.1) is a virtual short-circuit. The DGS is then grounded at both ends and hence it hardly affects the response of this mode. On the contrary, for CM operation the $A A^{\prime}$ plane behaves as a virtual open-circuit. It is then expected a strong disturbance of the CM response.

Let us first focus our attention on the role of the U-shaped DGSs when used below a differential line pair [see Fig.2(a)]. Each resonator can be modeled (CM excitation) as a parallel $L C$-tank circuit with a couple of electrically short lines connected at both sides [11] (see Fig.2). 


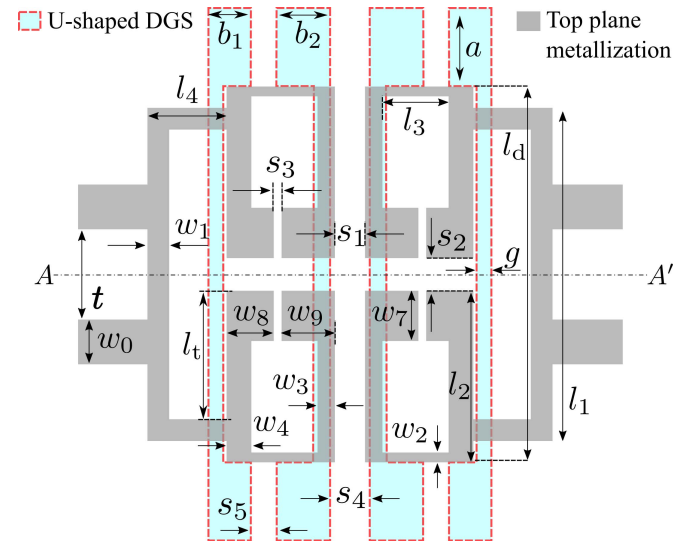

Fig. 1. Layout of the structure proposed for the implementation of a balanced dual-band bandpass filter. Dimensions (all in $\mathrm{mm}$ ) are: (i) top plane: $t=3.25$, $w_{0}=0.75, w_{1}=0.6, w_{2}=0.3, w_{3}=0.2, w_{4}=0.6, w_{7}=1.21, w_{8}=0.8$, $w_{9}=1.7, l_{1}=8.6, l_{2}=4.11, l_{3}=1.9, l_{4}=1.6, s_{1}=0.24, s_{2}=0.38$ and $s_{3}=0.2$; (ii) bottom plane: $b_{1}=1.13, b_{2}=1.56, a=2.85, l_{\mathrm{d}}=9.6$, $g=0.2, s_{4}=0.23$ and $s_{5}=0.2$.
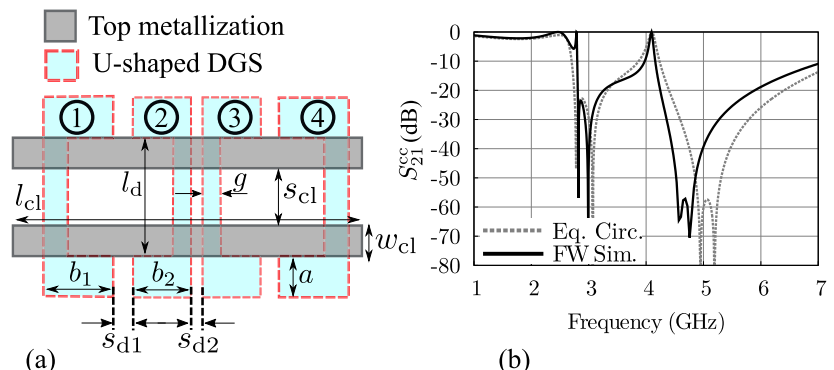

(a)

(b)

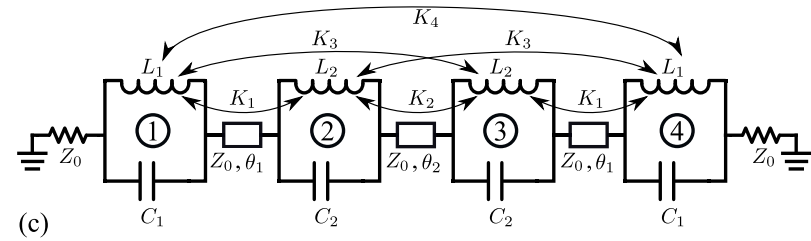

Fig. 2. (a) Differential lines with four coupled U-shaped DGSs (not to scale). (b) Full-wave and equivalent circuit simulations (CM operation). (c) Equivalent circuit for $\mathrm{CM}$ operation. Dimensions $(\mathrm{mm}): l_{\mathrm{cl}}=12$, $w_{\mathrm{cl}}=0.75, s_{\mathrm{cl}}=4.05, b_{1}=1.53, b_{2}=1.06, a=6.65, g=0.2, l_{\mathrm{d}}=5.6$, $s_{\mathrm{d} 1}=0.2$ and $s_{\mathrm{d} 2}=0.1$. Extracted electrical parameters from [11] are: $C_{1}=0.68 \mathrm{pF}, C_{2}=0.409 \mathrm{pF}, L_{1}=2.74 \mathrm{nH}, L_{2}=3.95 \mathrm{nH}, K_{1}=-0.228$, $K_{2}=0.05, K_{3}=-0.0172$ and $K_{4}=0.0012 . Z_{0}=50 \Omega, \theta_{1}=11.02^{\circ}$ and $\theta_{2}=0.69^{\circ}(@ 1 \mathrm{GHz})$.

A series-connected $L C$-tank resonator introduces a transmission zero (TZ) at its resonance frequency. This frequency can be allocated by selecting the values of $L$ and $C$ which, in turn, depend on the physical dimensions of the resonator, i.e., $b, a, g$, and $l_{\mathrm{d}}$ in Fig.2. The proposed slotted ground pattern is aimed to reject the CM within the two DM passbands. Hence, it is necessary to introduce several CM TZs in the proper locations. Several coupled U-shaped slot-like resonators are etched in the ground plane with this purpose. From network theory it is well known that any transmission line loaded with $n$ coupled resonators presents $n$ transmission zeros (even if the only considered couplings are those between adjacent resonators). Such zeros can be allocated in the frequencies of interest by properly choosing the values $L_{i}$ and $C_{i}(i=1,2)$ and the couplings between resonators. To illustrate this fact, Fig. 2 depicts an example of CM response when four U-shaped resonators are introduced below a simple pair of differential lines (CM rejection filtering). In this example, the substrate parameters are: dielectric constant $\varepsilon_{r}=5.8$, loss tangent $\tan \delta=0.0022$, and substrate thickness $h=0.508 \mathrm{~mm}$. The value of the lumped-elements, $L_{i}$ and $C_{i}(i=1,2)$, and the inductive coupling between resonators, $K_{i}(i=1 \ldots 4)$, for the configuration in Fig.2(a) have been extracted using the procedure reported in [11]. The simulated performance (full-wave and circuit-model simulations) is shown in Fig.2(b), where two CM rejection bands clearly appear due to the presence of the four U-shaped coupled resonators. It is important to mention that the nature of the coupling between resonators (i.e., electric, magnetic or mixed) has been determined following the methodology described in [14] and [15]. An example of the application of this DGS structure to the design of a balanced dual-band filter is given in the next section.

\section{EXAMPLE OF DUAL-BAND Filter DeSIGN}

\section{A. Conventional Filter}

Let us first consider the design of a dual-band balanced filter without the DGS structure (the same layout as in Fig.1 but with a solid ground plane). The guidelines given in [14] are followed. The procedure makes use of the external quality factors, $Q_{j}$, and the coupling coefficients, $M_{(1,2)}^{j}(j=1,2)$. For the proposed topology, the filter specs are: Butterworth of order $N=2$; center frequencies $f_{\mathrm{DM}^{(1)}}=2.5 \mathrm{GHz}$ and $f_{\mathrm{DM}}^{(2)}=5.6 \mathrm{GHz}$; fractional bandwidths $\Delta_{\mathrm{DM}}^{(1)}=8 \%$ and $\Delta_{\mathrm{DM}}^{(2)}=5 \%$. The same substrate as in the previous section is used. The required external quality factors and coupling coefficients result to be: $Q_{1}=17.67, Q_{2}=28.28$, $M_{1,2}^{(1)}=0.056$ and $M_{1,2}^{(2)}=0.035$. For the conventional filter, the external quality factor and coupling coefficient of the first DM passband are controlled by the tapping position, $t$, and the gap distance, $s_{1}$, respectively. Once $t$ and $s_{1}$ are obtained for the first passband, the external quality factor of the second passband is adjusted by choosing the appropriate value of $l_{\mathrm{t}}$. The required coupling coefficient is achieved by tuning the ratio $w_{3} / w_{4}$ [6], [10]. The obtained final dimensions (in $\mathrm{mm}$ ) are: $t=3.11, w_{0}=0.75, w_{1}=0.6, w_{2}=0.43, w_{3}=0.35$, $w_{4}=0.6, w_{7}=0.91, w_{8}=1.29, w_{9}=1.68, l_{1}=8.6$, $l_{2}=4.44, l_{3}=2.22, l_{4}=2.11, s_{1}=0.28, s_{2}=1.08$, and $s_{3}=0.2$.

\section{B. Filter With Embedded U-Shaped DGS Structure}

Once the conventional filter has been designed, the proposed DGS designed in section II, consisting of four U-shaped coupled resonators, is introduced in the ground plane, just below the filter layout. Since the CM signal must be rejected within the two differential passbands, the DGS pattern has been optimized so as to avoid $\mathrm{CM}$ propagation in the 2-3 GHz and 5-6 GHz bands. The physical parameters of the DGSs in section II have been used as initial guesses for the final optimization step. This process has been carried out with the help of ADS Momentum [13] (See Fig.1 for dimensions. Note please that some dimensions of the conventional filter have been slightly modified in the new structure in order to compensate for the small perturbations introduced by the DGS on the DM response). 
TABLE I

Comparison of Several Dual-Band Balanced Filters

\begin{tabular}{c|c|c|c|c} 
Ref. & $\begin{array}{c}f_{\mathrm{DM}}^{1,2} \\
(\mathrm{GHz})\end{array}$ & $\begin{array}{c}\mathrm{IL} \\
(\mathrm{dB})\end{array}$ & $\begin{array}{c}\text { Size } \\
\left(\lambda_{\mathrm{g}} \times \lambda_{\mathrm{g}}\right)\end{array}$ & $\begin{array}{c}\text { CMRR } @ \\
f_{\mathrm{DM}}^{1,2}(\mathrm{~dB})\end{array}$ \\
\hline \hline$[1]$ & $2.44 / 5.57$ & $1.78 / 2.53$ & $0.258 \times 0.592$ & $36.2 / 30.5$ \\
{$[2]$} & $2.44 / 5.25$ & $2.4 / 2.82$ & $0.420 \times 0.380$ & $52.6 / 39.2$ \\
{$[3]$} & $1.84 / 2.45$ & $2.20 / 2.60$ & $0.187 \times 0.285$ & $20.5 / 20.0$ \\
{$[4]$} & $2.46 / 5.56$ & $0.96 / 1.9$ & $0.314 \times 0.413$ & $36.2 / 31.1$ \\
{$[5]$} & $2.40 / 3.57$ & $0.87 / 1.90$ & $0.50 \times 0.20$ & $27.1 / 29.1$ \\
{$[6]$} & $2.50 / 5.27$ & $1.46 / 2.22$ & $0.262 \times 0.637$ & $38.5 / 22.8$ \\
{$[7]$} & $2.50 / 5.80$ & $0.77 / 1.56$ & $0.343 \times 0.133$ & $41.2 / 36.4$ \\
{$[8]$} & $9.23 / 14.05$ & $2.90 / 2.70$ & $2.70 \times 1.27$ & $45.3 / 38.7$ \\
{$[9]$} & $1.57 / 2.47$ & $0.53 / 0.72$ & $0.14 \times 0.23$ & $54.5 / 42.3$ \\
This & $2.50 / 5.60$ & $1.29 / 1.97$ & $0.153 \times 0.268$ & $34.7 / 24.1$ \\
\hline
\end{tabular}

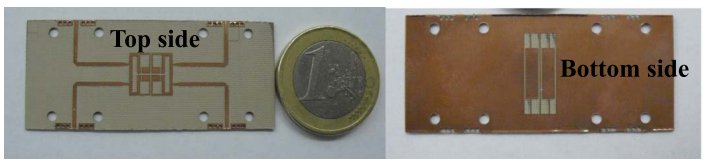

Fig. 3. Photograph of the fabricated prototype (top and bottom sides).

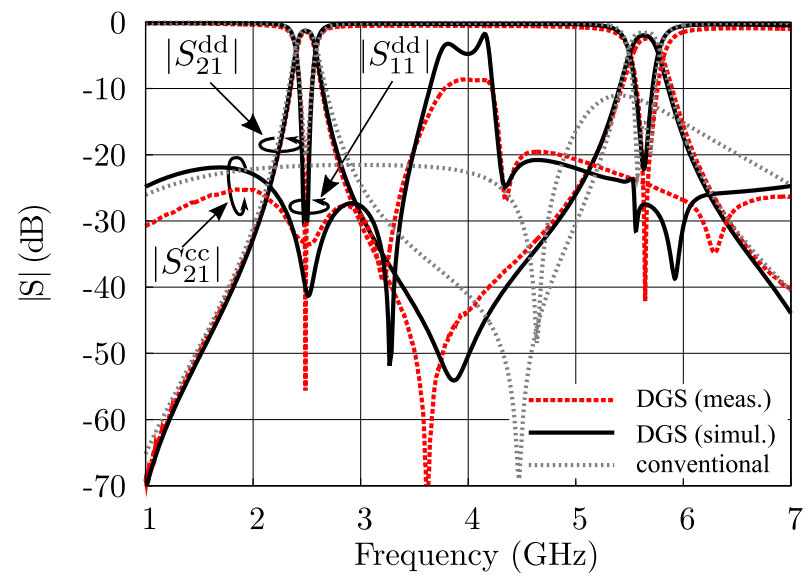

Fig. 4. Measured DM and CM responses for the DGS-based filter. $\mathrm{DM}$ and $\mathrm{CM}$ responses of the conventional filter have been included for comparison purposes. Simulations have been carried out with ADS-Momentum [13].

\section{Experimental Results}

In order to validate our proposal, a prototype of the DGS-based designed filter has been fabricated (see photo in Fig.3) and measured using an Agilent PNA-E8363B with a N4420B test-set extension. Simulated and measured DM and $\mathrm{CM}$ responses are shown in Fig.4. The CM and DM responses of the conventional filter (with solid ground plane) have been included for comparison. This figure clearly shows the CM rejection improvement as well as the good agreement between simulations and measurements for the novel filter. It can be appreciated the absence in the measurements of the simulated CM transmission zero located at approximately $5.5 \mathrm{GHz}$ (DGS filter). It has been verified that this TZ is very sensitive to the fabrication tolerances. However, even without that $\mathrm{TZ}, \mathrm{CM}$ rejection has been improved in about $15 \mathrm{~dB}$ when compared with the conventional case. A comparison with other contributions is given in Table I. Common-mode rejection ratio (CMRR) and electrical size are found to be competitive when compared with the other designs.

\section{CONCLUSION}

A new balanced dual-band bandpass filter based on embedded resonators and U-shaped DGS has been presented. An easy-to-follow step-by-step design procedure is described. The first step consists on the design of the conventional filter with solid ground plane. Next, four coupled U-shaped DGS resonators are introduced, which are symmetrically located below the filter area. The parameters of the U-shaped slots are set in order to get good CM rejection over the frequency ranges of interest, namely, the two differential passbands. Finally, some physical dimensions of the top-layer resonators are slightly tuned to compensate for the small effect of the ground plane slots. The proposed structure provides a significant improvement of the CMRR while does not deteriorate the integrity of the differential-mode response and does not increase the filter size.

\section{REFERENCES}

[1] J. Shi and Q. Xue, "Novel balanced dual-band bandpass filter using coupled stepped impedance-resonators," IEEE Microw. Wireless Compon. Lett., vol. 20, no. 1, pp. 19-21, Jan. 2010.

[2] C.-H. Lee, C.-I.-G. Hsu, and C.-C. Hsu, "Balanced dual-band BPF with stub-loaded SIRs for common-mode suppression," IEEE Microw. Wireless Compon. Lett., vol. 20, no. 2, pp. 70-72, Feb. 2010.

[3] J. Shi and Q. Xue, "Balanced bandpass filters using center-loaded halfwavelength resonators," IEEE Trans. Microw. Theory Techn., vol. 58, no. 4, pp. 970-977, Apr. 2010

[4] J. Shi and Q. Xue, "Dual-band and wide-stopband single-band balanced bandpass filter with high selectivity and common-mode suppression," IEEE Trans. Microw. Theory Techn., vol. 58, no. 8, pp. 2204-2212, Aug. 2010.

[5] Y.-H. Cho and S.-W. Yun, "Design of balanced dual-band bandpass filters using asymmetrical coupled lines," IEEE Trans. Microw. Theory Techn., vol. 61, no. 8, pp. 2814-2820, Aug. 2013.

[6] A. Fernández-Prieto et al., "Dual-band differential filter using broadband common-mode rejection artificial transmission line," Prog. Electromag. Res., vol. 139, pp. 779-797, Apr. 2013.

[7] F. Wei, Y. J. Guo, P. Y. Qin, and X. W. Shi, "Compact balanced dualand tri-band bandpass filters based on stub loaded resonators," IEEE Microw. Wireless Compon. Lett., vol. 25, no. 2, pp. 76-78, Feb. 2015.

[8] Y. Shen, H. Wang, W. Kang, and W. Wu, "Dual-band SIW differential bandpass filter with improved common-mode suppression," IEEE Microw. Wireless Compon. Lett., vol. 25, no. 2, pp. 100-102, Feb. 2015.

[9] F. Wei, P. Y. Qin, Y. J. Guo, C. Ding, and X. W. Shi, "Compact balanced dual- and tri-band BPFs based on coupled complementary split-ring resonators (C-CSRR)," IEEE Microw. Wireless Compon. Lett., vol. 26, no. 2, pp. 107-109, Feb. 2016.

[10] C.-Y. Chen, C.-Y. Hsu, and H.-R. Chuang, "Design of miniature planar dual-band filter using dual-feeding structures and embedded resonators," IEEE Microw. Wireless Compon. Lett., vol. 16, no. 12, pp. 669-671, Dec. 2006.

[11] S.-J. Wu, C.-H. Tsai, T.-L. Wu, and T. Itoh, "A novel wideband common-mode suppression filter for gigahertz differential signals using coupled patterned ground structures," IEEE Trans. Microw. Theory Techn., vol. 57, no. 4, pp. 848-855, Apr. 2009.

[12] Z. Zeng, Y. Zhuang, Z. Shen, Y. Yao, and X. Xiang, "A novel simple wideband common-mode suppression filter," IEICE Electron. Exp., vol. 11, no. 17, pp. 1-6, Sep. 2014.

[13] Keysight Technologies. (Jan. 2016). ADS-Momentum. [Online]. Available: http://www.keysight.com

[14] J. S. Hong, Microstrip Filters for RF/Microwave Applications, 2nd ed. New York, NY, USA: Wiley, 2011, ch. 7.

[15] J.-S. Hong and M. J. Lancaster, "Couplings of microstrip square openloop resonators for cross-coupled planar microwave filters," IEEE Trans. Microw. Theory Techn., vol. 44, no. 11, pp. 2099-2109, Nov. 1996. 